When less is more - implicit preference for incomplete bodies

\title{
in Xenomelia
}

Gianluca Macauda ${ }^{1}$, Robin Bekrater-Bodmann ${ }^{2}$, Peter Brugger ${ }^{1,3}$, Bigna Lenggenhager ${ }^{1,3}$ 


\begin{abstract}
Individuals with xenomelia identify with an amputated rather than with their physically complete, healthy body. They often mimic amputees and show a strong admiration of and sexual attraction towards them. Here we investigated for the first time empirically whether such unusual preference for amputated bodies is present also on an implicit level. Using the well-validated Implicit Association Test we show that individuals with xenomelia manifested a stronger implicit and explicit preference for amputated bodies stronger than a normallylimbed control group and a group of involuntary amputees did. Interestingly, the two latter groups did not differ in their implicit and explicit preference for complete versus amputated bodies. These findings are an important step in understanding how deeply rooted attitudes about a socially normative body appearance may be influenced by a developmentally disordered experience of one's own bodily self. We conclude that this first behavioral evidence to demonstrate a conflict of self-identification on an implicit level and this enriches current understandings of xenomelia as a primarily neurological disorder.
\end{abstract}

Key Words: Bodily Self, Social Self, Body Integrity Identity Disorder, Web experiment, Implicit Association Test 


\section{IAT IN XENOMELIA}

\section{Introduction}

The experience that we own our body is often taken for granted. Yet, the unity of body and self is surprisingly vulnerable (Brugger and Lenggenhager, 2014). A case in point is Body Integrity Identity Disorder (BIID) (First, 2005), also described as xenomelia ("foreign limb syn-

drome") (McGeoch et al., 2011), a condition which currently captures the attention of the popular media (Ananthaswamy, 2015) and that of scientific representatives (Ramachandran et al., 2009) and medical ethics (Ryan, 2008) alike.

Individuals with xenomelia experience their body as disturbingly "overcomplete": they feel that their true identity is that of an amputee and accordingly demand the right of elective limb amputation (Ryan, 2008). While previous research focused on xenomelia as a neurological condition in the narrow sense (Hilti et al., 2013; McGeoch et al., 2011), several associated signs point to significant social co-determinants (Brugger et al., 2013; Brugger and Lenggenhager, 2014). First, the desire for amputation is often triggered by encounters with amputees (Aoyama et al., 2012). Second, a profound admiration for amputees up to a sexual attraction towards incomplete bodies has been described in some individuals with xenomelia (De Preester, 2013; First, 2005) and third, affected persons frequently pretend in private or public to be amputees. The perception of one's own and another person's body are intimately linked (Schilder, 1935), and sensorimotor processes are influenced by observation and imitation of others (de Guzman et al., 2016; Tsakiris, 2016). Such shared neural mechanisms are modulated by interpersonal relations (e.g. Désy and Théoret, 2007), generally suggesting that the stronger they are, the more similar and positive another person is explicitly but also implicitly perceived and vice versa (Maister et al., 2013). While an overt behavioral preference for having an amputated body is a core aspect of xenomelia, it is unknown whether positive attitudes towards amputated bodies are also represented on a more implicit level. Here, we recruited individuals with xenomelia and two age- and sex- 


\section{IAT IN XENOMELIA}

matched groups of normally-limbed controls and involuntary limb amputees to investigate their implicit preference for an amputated body, as measured with a web based implicit association test (IAT) (Greenwald et al., 1998). We hypothesized that individuals with xenomelia compared to normally-limbed persons would show a stronger explicit preference for amputated bodies. More importantly, we predicted that unlike the normally-limbed controls, they would also show an implicit preference for amputated bodies. Similarly, Olson and colleagues (2015) found in children with Gender Dysphoria, a condition with which xenomelia shares several key features (First, 2005), that their explicit preferences for the expressed gender is also reflected in an implicit preference as measured by the IAT. For the comparison of individuals with xenomelia and the amputees, the available literature was not sufficient to develop directed hypotheses for the explicit and implicit preferences.

\section{Methods}

\section{Participants}

Participants with xenomelia were recruited over dedicated internet platforms and organizations and were thus self-designated individuals with xenomelia. Other psychiatric conditions could not strictly be excluded. Age- and sex- matched control participants with an amputation were recruited using the database created for a previous comprehensive study about body representation in amputees (e.g. Bekrater-Bodmann et al., 2015).

Our original sample of individuals with xenomelia consisted of 45 participants. However, to reduce heterogeneity, we excluded nine individuals who already underwent an amputation. Moreover, we excluded two further participants who desired not amputation, but paralysis. Thirty-four individuals with self-declared amputation desire for one or both legs, 35 lower-limb amputees, and 35 normally-limbed controls were finally included in the study. Most involuntary amputees underwent an amputation after traffic or work accidents. A Bayesian ANOVA revealed 


\section{IAT IN XENOMELIA}

weak evidence for no age differences between the three groups $\left(\mathrm{BF}_{01}=2.58\right)$ in comparison to a model including an age effect. A detailed description of the participants is listed in table 1 .

The study was approved by the Cantonal Ethics Committee of Zurich and conducted according to the ethical standards of the Declaration of Helsinki.

*Insert Table 1 around here*

\section{General procedure}

Participants were asked to perform the experiment online on a computer with a keyboard and a mouse in a calm moment without any disturbances, and in one session. The full experiment included the IAT, the explicit questions targeting body preference, some group-specific questions and the Body Image Task (Fuentes et al., 2013a, 2013b). The latter is not reported here, as the number of participants was too low (only 14 individuals with xenomelia completed the task) and an analysis of the data would not have allowed any reasonable interpretation. All testing took about thirty minutes.

\section{IAT}

To measure the participants' strength of automatic association between the concepts of a 'complete' or 'amputated body' and its positive and negative valence, we modified an existing open source and JavaScript based IAT (https://github.com/winteram/IAT). The IAT consisted of 20 drawn pictures of normally-limbed people (concept of complete body) or people with one missing leg (concept of amputated body) paired with words associated with positive (e.g. love, peaceful) or negative (e.g. war, horrible) concepts (for a more detailed description see Fig. 1 and its legend). The participants' bias towards an incomplete or complete body was measured by the response time and accuracy of categorizing the pictures with a positive concept relative to a negative concept. The IAT consisted of seven blocks (Greenwald et al., 2003),. All stimuli were displayed in the middle of a central white rectangle with the dimensions of $540 \times 540$ pixels (Fig. 


\section{IAT IN XENOMELIA}

2a). The stimulus remained on the screen until a response was given. Each block started with detailed instructions. Participants were instructed to respond as fast and accurately as possible with the keys 'e' and ' $i$ ' on the keyboard. After an incorrect response a red ' $\mathrm{X}$ ' popped up immediately. An incorrect response was defined as the misclassification of a picture or a word. Responses longer than 10 seconds were excluded and latencies of incorrect responses were replaced with the block mean and added a penalty of $600 \mathrm{~ms}$, according to the improved algorithm to calculate the IAT value proposed by Greenwald and colleagues (2003). The IAT value was calculated with the improved scoring algorithm: The mean difference in reaction time between the incongruent and congruent blocks was divided by the pooled standard deviation (SD) of all correct trials so that positive IAT scores indicate an implicit preference for complete bodies and negative IAT scores indicate an implicit preference for amputated bodies.

$* * *$ Insert Figure 1 around here $* * *$

\section{Explicit attitude}

Participants were also asked to rate the valence of the concepts of 'complete bodies' and 'amputated bodies' on a rating scale from 0 to 20 . The difference between these two ratings was computed and normalized so that it ranged from -1 (maximally positive attitude towards amputated bodies, accordingly, and maximally negative attitude towards complete bodies) to 1 (maximally negative attitude towards amputated bodies and, accordingly, maximally positive attitude towards complete bodies). One amputee did not rate the valence of "complete body" and his normalized difference value was thus excluded from further analyses.

\section{Group specific questions}

To quantify core aspects of xenomelia, we adopted an online version of the Zurich Xenomelia Scale (ZXS) for the recruited xenomelia group. The ZXS is a questionnaire that quantifies three essential aspects of xenomelia ('pure amputation desire', 'erotic attraction' and 'pre- 


\section{IAT IN XENOMELIA}

tending behavior') on a scale from 1 to 6 and is described in more detail elsewhere (Aoyama et al., 2012).

They were also asked to indicate which $\operatorname{limb}(\mathrm{s})$ their amputation desire is directed to. Moreover, they were asked whether they already underwent an amputation and to indicate their age when first becoming aware of the desire. They were also asked to assess the subjective probability of an amputation of the undesired limb within a year on a scale from 0 to 20 .

Amputees were asked how strongly they experienced a phantom of their amputated limb on a numerical rating scale from 0 (no phantom) to 20 (very strong), how strong their phantom pain was from 0 (no phantom pain) to 20 (very strong), and how often they used a prosthesis from 0 (never) to 20 (very often). Those values were used for further explorative correlational analyses.

\section{Body representation in dreams}

Based on previous reviews and finding regarding recalled body representation in dreams in amputees (Bekrater-Bodmann et al., 2015; Brugger, 2008), participants were asked to rate how often they recalled their body during dreams as complete or incomplete on a numerical rating scale from 0 (never) to 20 (always). The difference between these two ratings was computed and normalized so that it ranged from -1 (very often incomplete and never complete) to 1 (very often complete and never incomplete). Those normalized values were used for further explorative correlational analyses.

\section{Statistical Analysis}

All statistical analyses were performed using the statistical language R (R Core Team, 2013). A Bayesian data analysis approach was chosen as it allows expressing statistical evidence for a defined compared to a null hypothesis using BFs (the ratio of the data's likelihood given two competing hypotheses) (Rouder et al., 2009). For group comparisons, two-sampled Bayesian 


\section{IAT IN XENOMELIA}

Jeffreys-Zellner-Siow (JZS) t-tests with default-scaled Cauchy priors $(r=.707)$ were calculated using the BayesFactor package for R (Morey and Rouder, 2015). The null model is defined as no difference between two groups. According to Jeffreys (1961), a BF above 3 yields substantial evidence for one of the two defined models, in our case for the null model. A BF below 1/3 provides substantial evidence for the alternative model. The credible interval (CI) is defined as $95 \%$ interval of the posterior density of the effect size parameter $\delta$.

For further correlational explorative analyses, correlation coefficients and BFs were calculated for undirected correlations using JASP (Love et al., 2015) and a beta prior width of 1 . The posterior distributions of the most interesting correlations were described in more detail using the BayesMed package implemented in R (Nuijten et al., 2015). This package provides functions for default Bayesian hypothesis tests for correlations using a JZS prior set-up. The posterior distributions were obtained from 10,000 Monte Carlo Markov Chain iterations and 1,000 burn-in iterations discarded at the beginning.

\section{Results}

\section{IAT}

Results of the IAT are shown in Figure 2C. Data analysis (see SOM for additional correlations and an analogous analysis using null hypothesis testing $)$ suggests weak evidence $\left(\mathrm{BF}_{01}=\right.$ .74 , credible interval (CI) for $\delta=[-.66,-.0005])$ for an implicit preference for the amputated body in the xenomelia group (IAT score mean $=-0.18$ ), whereas both amputees $($ IAT mean $=.85$, $\mathrm{BF}_{01}=2.85 \times 10^{-10}, \mathrm{CI}$ for $\left.\delta=[1.21,2.28]\right)$ and the normally-limbed controls (IAT mean $=.86$, $\mathrm{BF}_{01}=3.82 \times 10^{-13}, \mathrm{CI}$ for $\left.\delta=[1.62,2.89]\right)$ show a strong implicit preference for the complete body. Two sampled Bayesian t-tests of the IAT score revealed a relatively stronger preference towards amputated bodies in the xenomelia group as compared to both the amputees $\left(\mathrm{BF}_{01}=1.06\right.$ $\times 10^{-10}, \mathrm{CI}$ for $\left.\delta=[-2.69,-1.46]\right)$ and the normally-limbed controls $\left(\mathrm{BF}_{01}=1.60 \times 10^{-12}, \mathrm{CI}\right.$ for $\delta$ 


\section{IAT IN XENOMELIA}

$=[-2.96,-1.70])$. There is substantial evidence for the model assuming no difference in the latter two groups' implicit preferences $\left(\mathrm{BF}_{01}=4.05, \mathrm{CI}\right.$ for $\left.\delta=[-.43, .43]\right)$.

\section{Explicit Values}

Data analysis for the explicit preferences shows strong evidence $\left(\mathrm{BF}_{01}=1.03 \times 10^{-12}, \mathrm{CI}\right.$ for $\delta=[-2.95,-1.61])$ for an explicit preference for the amputated body in the xenomelia group $($ mean $=-.72)($ Fig. $2 \mathrm{~B})$, whereas both amputees $\left(\right.$ mean $=.65, \mathrm{BF}_{01}=9.85 \times 10^{-11}, \mathrm{CI}$ for $\delta=$ $[1.30,2.48]$ ) and the normally-limbed controls (mean $=.66, \mathrm{BF}_{01}=2.06 \times 10^{-8}, \mathrm{CI}$ for $\delta=[.96$, 1.94]) show a strong explicit preference for the complete body (see Figure 2D). The xenomelia group preferred the amputated body more than the amputees $\left(\mathrm{BF}_{01}=1.39 \times 10^{-23}, \mathrm{CI}\right.$ for $\delta=[-$ $4.9,-3.28])$ and the normally-limbed controls $\left(\mathrm{BF}_{01}=1.82 \times 10^{-20}\right.$, CI for $\left.\delta=[-4.31,-2.82]\right)$, while for the two latter groups there is substantial evidence for the model postulating no difference $\left(\mathrm{BF}_{01}=4.03, \mathrm{CI}\right.$ for $\left.\delta=[-.43, .42]\right)$.

*** Insert Figure 2 around here ***

\section{Correlations}

Explorative correlations are shown in Supplementary Tables 1-3. Using the JSZ prior setup implemented in the BayesMed package, the posterior distribution of the correlation coefficient for the duration of amputation desire and the body representation in dreams in the xenomelia group yielded a mean correlation of -.47 with a CI ranging from -.83 to -.10 and posterior probability of a correlation of .78. Likewise, the posterior distribution of the correlation coefficient for the relative explicit preference and the pure amputation score in the ZXS in the xenomelia group showed a mean correlation of -.48 with a CI ranging from -.78 to -.17 and posterior probability of a correlation of .92 . 


\section{IAT IN XENOMELIA}

In the amputees, there was a positive correlation between phantom pain strength and the IAT score with a mean correlation coefficient of .49 , a CI ranging from 0.19 to .78 and a posterior probability of a correlation of .94 .

\section{Discussion}

The present study investigated explicit and, more importantly, implicit attitudes towards complete and amputated bodies in individuals suffering from xenomelia, involuntary amputees, and normally-limbed controls. It is thus the first study directly comparing a rather large and homogenous sample of individuals with xenomelia with participants actually suffering from amputation, with both groups having their lower limbs affected.

While amputees and normally-limbed controls showed strong implicit and explicit preferences for complete bodies, which is in line with people's general attitude towards disabled individuals (e.g. Wilson and Scior, 2014), the IAT scores from individuals with xenomelia differed strongly. The latter group showed a strong explicit and a weak implicit preference for amputated bodies. This is the first behavioral evidence for a conflict of self-identification in xenomelia on an implicit level, as affected individuals appear to prefer the bodily state of an out-group that does not physically match its membership criteria. Plausibly, the identification with or attraction towards amputees was strong enough to overcome negative biases and connotations typically associated with amputees. Yet, from the current results it is not possible to deduce whether there is a causal relationship between the implicit preference for impaired bodies and the persistent mimicking of the amputees' behavior. Blurred self-other-boundaries induced through own-body related multisensory conflicts have shown to influence implicit attitudes (Maister et al., 2015) and vice versa (Bufalari et al., 2014), indicating that an altered representation of the body might contribute to the present effects. The weak preference for amputated bodies is even more remarkable, when considering that the involuntary amputees showed the same negative bias towards 


\section{IAT IN XENOMELIA}

amputees as the normally-limbed controls did. This finding is in line with recent evidence from spinal cord injury patients, who continue to show negative implicit attitudes towards their own ingroup despite a change in explicit preference (Galli et al., 2015). The positive correlation between the IAT score and phantom pain but not phantom sensations experienced by the amputees in the present sample (see SOM for the explorative correlations), could suggest that pain keeps the negative connotations upright. Interestingly, explicit and implicit preferences in our study were unrelated in all three groups. Corresponding to this dissociation, the pure amputation subscale of the ZXS was associated with explicit but not implicit body preferences. Similarly, a literature review on attitudes towards individuals with disabilities concluded that there is weak to no association between explicit and implicit measures (Wilson and Scior, 2014). A low correspondence between explicit and implicit measures has been shown to be associated with four factors (Nosek, 2005): a) self-representation, which describes the modification of an explicit preference for personal or social reasons, b) evaluative strength, which predicts higher associations for personally important and frequently emerging contexts, c) dimensionality, with unipolar categories causing lower associations compared to bipolar ones, and d) distinctiveness, which characterizes the perceived incongruence between one's own and societal norms. We can only speculate about which factor might have driven the missing relationship between implicit and explicit measures in the present study. However, since all groups show this missing association to a comparable degree, it is likely that the dimensionality behind preferences for intact and amputated bodies is rather unipolar than bipolar, indicating that an individual with xenomelia could prefer amputated bodies while simultaneously not rejecting intact bodies. Furthermore, considering the duration of the desire in the xenomelia group (in our sample on average more than 35 years), it is likely that higher-order cognitions based on long-lasting experiences overbuild and shape explicit measures. 


\section{IAT IN XENOMELIA}

The fact that the individuals with xenomelia behave strikingly different from normally limbed controls and amputees, stresses the importance of considering social factors in current scientific models of xenomelia. A similar implicit preference for the desired rather than the physical body state has only recently been shown empirically in children with Gender Dysphoria (Olson et al., 2015), a condition clinically related to xenomelia, but socially more generally sanctioned. Our findings corroborate the importance of implicit measures towards body states in conditions of a mismatch between physical realization and desired ideal of one's body. The comparison between individuals with xenomelia and participants suffering from an involuntary amputation provides insights into the relation between one's physical body and an internally stored body model. Amputees showed a remarkably preserved preference for intact bodies, even decades after amputation. This finding is confirmed by a missing relationship between time since amputation and implicit attitude towards the intactness of a body, as it indicates that the perception and evaluation of physical attributes in in- and outgroups might be relatively stable. For social evaluation, amputees might draw back on an internally stored body model, which finds perceptual expression in the fact that more than $80 \%$ of limb amputees report phantom limb awareness (Giummarra et al., 2010). In contrast and while physically intact, individuals suffering from xenomelia report perceived foreignness for the affected body part (Hilti et al., 2013). If the present results rely on a stored body model independent of the physical body, the implicit preference for amputated bodies in xenomelia might be opposite to what is observed in amputees. The desire for amputation as well as pretending behavior in xenomelia might not only reflect the need to feel physically 'intact', but also to belong to a group that is more similar to one's individual body representation. Prospective studies have to investigate how exactly an internally stored body model may shape social cognition. 


\section{IAT IN XENOMELIA}

\section{Limitations and Outlook}

The web-based nature of the study allows to collect data of a number of participants that would be unthinkable in a lab environment. At the same time, this procedure is accompanied by some disadvantages: The participation of the study is based on the self-diagnosis of xenomelia. Although we have control measurements such as the ZXS and the precise description of the demarcation line on the unwanted limb that favor the assumption of the participants' authenticity, there was no personal interview with detailed questions. We may add, however, that there are currently no established clinical diagnostic guidelines for diagnosing xenomelia.

The explicit measure we used relies entirely on two visual analog scales and its validity is thus barely comparable to the well-validated and reliable IAT. Because of their reliability, implicit measures such as the IAT offer an interesting addition to the future assessment of xenomelia, maybe even in a clinical context. At the same time, comparable explicit measures have to be developed further.

Individuals with xenomelia often report a very early onset of their desire. Thus, even more insight into the cognitive patterns of xenomelia could be gained from a comparison with individuals with congenital absence of a limb as it might sketch out developmental aspects of xenomelia (Hilti and Brugger, 2010). Such a comparison may elucidate some basic questions of both physical and representational integrity.

As the current study solely focused on the implicit attitude towards amputated bodies, the underlying neural mechanisms of such cognitive processes are still unknown. Neural correlates of the IAT (see e.g. Schiller et al., 2016) are of high interest as they would offer a more direct insight into the interaction of neurological and social aspects of. 


\section{References}

Ananthaswamy, A., 2015. The Man Who Wasn't There: Investigations into the Strange New Science of the Self. Penguin Publishing Group, New York.

Aoyama, A., Krummenacher, P., Palla, A., Hilti, L.M., Brugger, P., 2012. Impaired SpatialTemporal Integration of Touch in Xenomelia (Body Integrity Identity Disorder). Spat. Cogn. Comput. 12, 96-110. doi:10.1080/13875868.2011.603773

Bekrater-Bodmann, R., Schredl, M., Diers, M., Reinhard, I., Foell, J., Trojan, J., Fuchs, X., Flor, H., 2015. Post-Amputation Pain Is Associated with the Recall of an Impaired Body Representation in Dreams-Results from a Nation-Wide Survey on Limb Amputees. PLoS ONE 10, e0119552. doi:10.1371/journal.pone.0119552

Brugger, P., 2008. The phantom limb in dreams. Conscious. Cogn. 17, 1272-1278-1280. doi:10.1016/j.concog.2008.01.005

Brugger, P., Lenggenhager, B., 2014. The bodily self and its disorders: neurological, psychological and social aspects. Curr. Opin. Neurol. doi:10.1097/WCO.0000000000000151

Brugger, P., Lenggenhager, B., Giummarra, M.J., 2013. Xenomelia: A Social Neuroscience View of Altered Bodily Self-Consciousness. Front. Psychol. 4. doi:10.3389/fpsyg.2013.00204

Bufalari, I., Lenggenhager, B., Porciello, G., Serra Holmes, B., Aglioti, S.M., 2014. Enfacing others but only if they are nice to you. Front. Behav. Neurosci. 8 . doi:10.3389/fnbeh.2014.00102

de Guzman, M., Bird, G., Banissy, M.J., Catmur, C., 2016. Self-other control processes in social cognition: from imitation to empathy. Philos. Trans. R. Soc. Lond. B. Biol. Sci. 371, 20150079. doi:10.1098/rstb.2015.0079

De Preester, H., 2013. Merleau-Ponty's sexual schema and the sexual component of body integrity identity disorder. Med. Health Care Philos. 16, 171-184. doi:10.1007/s11019-0119367-3

Désy, M.-C., Théoret, H., 2007. Modulation of Motor Cortex Excitability by Physical Similarity with an Observed Hand Action. PLoS ONE 2, e971. doi:10.1371/journal.pone.0000971

First, M.B., 2005. Desire for amputation of a limb: paraphilia, psychosis, or a new type of identity disorder. Psychol. Med. 35, 919-928.

Fuentes, C.T., Longo, M.R., Haggard, P., 2013a. Body image distortions in healthy adults. Acta Psychol. (Amst.) 144, 344-351. doi:10.1016/j.actpsy.2013.06.012

Fuentes, C.T., Pazzaglia, M., Longo, M.R., Scivoletto, G., Haggard, P., 2013b. Body image distortions following spinal cord injury. J. Neurol. Neurosurg. Psychiatry 84, 201-207. doi:10.1136/jnnp-2012-304001

Galli, G., Lenggenhager, B., Scivoletto, G., Molinari, M., Pazzaglia, M., 2015. Don’t look at my wheelchair! The plasticity of longlasting prejudice. Med. Educ. 49, 1239-1247. doi:10.1111/medu.12834

Giummarra, M.J., Georgiou-Karistianis, N., Nicholls, M.E., Gibson, S.J., Chou, M., Bradshaw, J.L., 2010. Corporeal awareness and proprioceptive sense of the phantom. Br. J. Psychol. 101, 791-808.

Greenwald, A.G., McGhee, D.E., Schwartz, J.L., 1998. Measuring individual differences in implicit cognition: the implicit association test. J. Pers. Soc. Psychol. 74, 1464-1480.

Greenwald, A.G., Nosek, B.A., Banaji, M.R., 2003. Understanding and using the implicit association test: I. An improved scoring algorithm. J. Pers. Soc. Psychol. 85, 197-216.

Hilti, L.M., Brugger, P., 2010. Incarnation and animation: physical versus representational deficits of body integrity. Exp. Brain Res. 204, 315-326. doi:10.1007/s00221-009-2043-7 


\section{IAT IN XENOMELIA}

Hilti, L.M., Hänggi, J., Vitacco, D.A., Kraemer, B., Palla, A., Luechinger, R., Jäncke, L., Brugger, P., 2013. The desire for healthy limb amputation: structural brain correlates and clinical features of xenomelia. Brain J. Neurol. 136, 318-329. doi:10.1093/brain/aws316

Jeffreys, H., 1961. Theory of Probability (3rd edt.) Oxford University Press.

Love, J., Selker, R., Marsman, M., Jamil, T., Dropmann, D., Verhagen, A.J., Ly, A., Gronau, Q.F., Smira, M., Epskamp, S., Matzke, D., Wild, A., Rouder, J.N., Morey, R.D., Wagenmakers, E.-J., 2015. JASP.

Maister, L., Sebanz, N., Knoblich, G., Tsakiris, M., 2013. Experiencing ownership over a darkskinned body reduces implicit racial bias. Cognition 128, 170-178. doi:10.1016/j.cognition.2013.04.002

Maister, L., Slater, M., Sanchez-Vives, M.V., Tsakiris, M., 2015. Changing bodies changes minds: owning another body affects social cognition. Trends Cogn. Sci. 19, 6-12. doi:10.1016/j.tics.2014.11.001

McGeoch, P.D., Brang, D., Song, T., Lee, R.R., Huang, M., Ramachandran, V.S., 2011. Xenomelia: a new right parietal lobe syndrome. J. Neurol. Neurosurg. Psychiatry 82, 1314-1319. doi:10.1136/jnnp-2011-300224

Morey, R.D., Rouder, J.N., 2015. BayesFactor: Computation of Bayes Factors for Common Designs.

Nosek, B.A., 2005. Moderators of the relationship between implicit and explicit evaluation. J. Exp. Psychol. Gen. 134, 565.

Nuijten, M.B., Wetzels, R., Matzke, D., Dolan, C.V., Wagenmakers, E.-J., 2015. A default Bayesian hypothesis test for mediation. Behav. Res. Methods 47, 85-97. doi:10.3758/s 13428-014-0470-2

Olson, K.R., Key, A.C., Eaton, N.R., 2015. Gender Cognition in Transgender Children. Psychol. Sci. 26, 467-474. doi:10.1177/0956797614568156

R Core Team, 2013. R: A Language and Environment for Statistical Computing. Vienna, Austria.

Ramachandran, V.S., Brang, D., McGeoch, P.D., Rosar, W., 2009. Sexual and food preference in apotemnophilia and anorexia: interactions between "beliefs" and "needs" regulated by two-way connections between body image and limbic structures. Perception 38, 775-777.

Rouder, J.N., Speckman, P.L., Sun, D., Morey, R.D., Iverson, G., 2009. Bayesian t tests for accepting and rejecting the null hypothesis. Psychon. Bull. Rev. 16, 225-237. doi:10.3758/PBR.16.2.225

Ryan, C.J., 2008. Out on a Limb: The Ethical Management of Body Integrity Identity Disorder. Neuroethics 2, 21-33. doi:10.1007/s12152-008-9026-4

Schilder, P., 1935. The image and appearance of the human body. Kegan Paul, Oxford, England.

Schiller, B., Gianotti, L.R.R., Baumgartner, T., Nash, K., Koenig, T., Knoch, D., 2016. Clocking the social mind by identifying mental processes in the IAT with electrical neuroimaging. Proc. Natl. Acad. Sci. 113, 2786-2791. doi:10.1073/pnas.1515828113

Tsakiris, M., 2016. The multisensory basis of the self: From body to identity to others. Q. J. Exp. Psychol. 0, 1-13. doi:10.1080/17470218.2016.1181768

Wilson, M.C., Scior, K., 2014. Attitudes towards individuals with disabilities as measured by the implicit association test: a literature review. Res. Dev. Disabil. 35, 294-321. doi:10.1016/j.ridd.2013.11.003 


\section{IAT IN XENOMELIA}

\section{Figure 1}

Fig. 1. A depiction of the seven blocks of the adapted online IAT. In the first two blocks, pictures and words were presented separately each 20 times and had to be classified as either positive/negative (words) or amputated/complete body (pictures) with the keys 'e' for positive and ' $i$ ' for negative concepts in block one and similarly in block one with the keys ' $e$ ' for complete bodies and ' $i$ ' for amputated bodies. In blocks three and four, the concepts of complete bodies and positive words, respectively were presented on the left (key ' $\mathrm{e}$ ' had to be pressed) while amputated bodies or negative words were shown on the right (key 'i' had to be pressed). Block three and four consisted of 80 trials in total. In these blocks, the association strength between the concepts 'good' and 'body complete'/'bad' and 'body amputated' were measured. In block five, pictures had to be categorized but with the response to complete (right, key 'i') and amputated bodies (left, key 'e') switched. In blocks six and seven, amputated bodies or positive words were presented on the left side (key 'e'), while complete bodies or negative words were presented on the right (key ' $i$ '). Those last two blocks consisted of 80 trials in total as well. In these blocks the association strength between the concepts 'bad' and 'body complete'/'good' and 'body amputated' were measured. 


\section{IAT IN XENOMELIA}

\section{Figure 2}

Fig. 2. Illustration of methods (upper panel) and results (lower panel) for the implicit measure of preferences (left) and for the explicit measure of preference (right). (a) A sample picture of an amputated body presented in the IAT to evaluate the implicit attitude towards complete and amputated bodies. (b) The two Visual Analog Scales used to measure the explicit attitudes towards complete and amputated bodies. (c) The IAT scores in the three tested groups as boxplots; positive values represent an implicit preference for complete bodies over amputated bodies. (d) The normalized differences of the explicit attitudes as boxplots; positive values represent an explicit preference for complete bodies. 


\begin{tabular}{|c|c|c|c|c|c|c|c|c|}
\hline & Age (in years) & Sex & $\begin{array}{r}\text { Afflicted lower } \\
\text { limb }\end{array}$ & $\begin{array}{r}\text { Duration of Amputation desire } \\
\text { / Time since amputation (in } \\
\text { years) }\end{array}$ & $\begin{array}{r}\text { ZXS Pure } \\
\text { Amputation (1 - 6) }\end{array}$ & $\begin{array}{r}\text { ZXS Erotic } \\
\text { Attraction (1 - 6) }\end{array}$ & $\begin{array}{l}\text { ZXS Pretending } \\
\text { Behavior (1 - 6) }\end{array}$ & $\begin{array}{l}\text { Subjective probability } \\
\text { of amputation }(0-20)\end{array}$ \\
\hline \multirow[t]{3}{*}{ Xenomelia } & $45.32( \pm 11.52)$ & Male $=32$ & Left $=19$ & $35.88( \pm 14.38)$ & $5.33( \pm 0.90)$ & $4.64( \pm 1.01)$ & $3.84( \pm 1.01)$ & $8.18( \pm 4.62)$ \\
\hline & Range $=28-77$ & Female $=2$ & Right $=8$ & Range $=6-67$ & Range $=2.5-6$ & Range $=2.75-6$ & Range $=1.5-5.25$ & Range $=2-16$ \\
\hline & & & Both $=7$ & & & & & \\
\hline \multirow[t]{2}{*}{ Amputees } & $50.97( \pm 9.11)$ & Male $=32$ & Left $=23$ & $23.74( \pm 11.38)$ & - & - & - & - \\
\hline & Range $=31-84$ & Female $=3$ & Right $=11$ & Range $=2-44$ & & & & \\
\hline
\end{tabular}

Table 1. Descriptives of the participants 
Both $=1$

Normally- $\quad 46.26( \pm 17.47) \quad$ Male $=30$

limbed $\quad$ Range $=21-72 \quad$ Female $=5$

For parametric data means and standard deviations (in brackets) are presented. ZXS = Zurich Xenomelia Scale 
